\title{
Obese Subjects with Non-Alcoholic Fatty Liver Disease Have a Higher Risk of Thyroid Dysfunction
}

\author{
Minyoung Kim ${ }^{1}$, Soo Kyoung Kim ${ }^{1,2}$, Jaehoon Jung 2,3 \\ ${ }^{I}$ Department of Internal Medicine, Gyeongsang National University Hospital, Gyeongsang National University School of \\ Medicine, Jinju, Korea \\ ${ }^{2}$ Institute of Health Sciences, Gyeongsang National University Hospital, Gyeongsang National University School of Med- \\ icine, Jinju, Korea \\ ${ }^{3}$ Department of Internal Medicine, Gyeongsang National University Changwon Hospital, Gyeongsang National University \\ School of Medicine, Jinju, Korea
}

Objectives: The effects of obesity on thyroid function have not been well established. The aim of this study was to investigate the effects of body mass index (BMI) and/or non-alcoholic fatty liver disease (NAFLD) on thyroid function.

Methods: A retrospective longitudinal analysis was conducted among subjects who underwent comprehensive health check-ups at least four times between 2008 and 2017. Thyroid function was investigated according to BMI or presence of NAFLD at the end of follow-up. The subjects were divided into four groups: control $(n=216)$, subjects with obese ( $\mathrm{n}$ $=94)$, subjects with NAFLD $(n=48)$, and subjects with obese + NAFLD $(n=93)$. Obesity was defined as BMI $\geq 25$ $\mathrm{kg} / \mathrm{m}^{2}$.

Results: During the mean follow-up of 6.8 years ( $6.8 \pm 1.2$ years), 42 of the 451 subjects $(9.3 \%)$ had subclinical hypothyroidism $(\mathrm{SCH})$ but no subjects developed overt hypothyroidism. In multivariate Cox proportional hazard analysis, after adjustment for age, sex, smoking, and baseline thyroid stimulating hormone level, obese subjects with NAFLD had a higher risk of $\mathrm{SCH}$ than the control group.

Conclusions: The obese subjects with NAFLD had a higher risk for SCH in the future.

Key Words: Body mass index, Hypothyroidism, Non-alcoholic fatty liver disease

With the increase in obesity, non-alcoholic fatty liver disease (NAFLD) has emerged as the most common liver disease and leading cause of cryptogenic cirrhosis worldwide. ${ }^{1,2}$ The prevalence of NAFLD is higher in subjects with subclinical and overt hypothyroidism. ${ }^{3}$ Thyroid hormones play an important role in energy expenditure, and thyroid dysfunction is associated with obesity. ${ }^{4-6}$ Overt hypothyroidism and subclinical hypothyroidism $(\mathrm{SCH})$ are associated with obesity and a high body mass index (BMI). ${ }^{4}$ Even in the normal range of thyroid function, there are associations among thyroid function, BMI, and fat distribution. ${ }^{5,6}$

However, recent studies indicated that thyroid stimulating hormone (TSH) levels could change

Corresponding Author: Jaehoon Jung, Department of Internal Medicine, Gyeongsang National University Changwon Hospital, 11 Samjeongja-ro, Seongsan-gu, Changwon-si 51472, Korea

Received: Jul. 23, 2019

Tel: +82-55-214-3740 Fax: +82-55-214-1031 E-mail: taesikjung@gmail.com

Revised: Jul. 23, 2019

\section{(9) (1) (2)}

Accepted: Oct. 03, 2019

Articles published in Kosin Medical Journal are open-access, distributed under the terms of the Creative Commons Attribution Non-Commercial License (http://creativecommons.org/licenses/by-nc/4.0/) which permits unrestricted non-commercial use, distribution, and reproduction in any medium, provided the original work is properly cited. 
secondary to obesity. ${ }^{7}$ Steatosis can cause cellular dysfunction when it accumulates in the nonadipose tissues of subject with obesity. For example, lipid accumulation in pancreatic betacells can cause beta-cell dysfunction. ${ }^{8}$ One study suggested that thyroid dysfunction may be associated with thyroid follicular cell steatosis and interfollicular fat accumulation. ${ }^{9}$ Furthermore, obesity could influence the hypothalamus-pituitary-thyroid (HPT) axis. ${ }^{10,11}$ TSH levels are elevated in obese subjects, and this is not accompanied by circulation thyroid antibody in most cases. They suggested that SCH may not indicate true hypothyroidism. ${ }^{12}$ Thus, the casual relationship between thyroid function and obesity is controversial, and few studies have assessed the relationship between thyroid function and obesity and steatosis. ${ }^{13-15}$ Therefore, the aim of our study was to investigate the effect of obesity and/or the presence of NAFLD (as a marker of steatosis in non-adipose tissue) on thyroid function.

\section{MATERIALS AND METHODS}

\section{Study design and subjects}

This study included 451 subjects who underwent a medical checkup at the health promotion center of Gyeongsang National University Hospital between 2008 and 2017. Many people undergo comprehensive health check-ups and some proportion of these individuals have a medical check-up annually or biannually. Initial data were obtained from 593 individuals who partic- ipated in comprehensive health check-ups at least four times between January 2008 and December 2017. Data from the first visit served as baseline data, and 142 subjects were excluded for the following reasons, all of which were based on data obtained at the initial visit: 1) known thyroid disease or abnormal thyroid function test (TSH $>10$ $\mathrm{mIU} / \mathrm{L} ; \mathrm{n}=31) ; 2)$ hepatitis $\mathrm{B}$ or $\mathrm{C}(\mathrm{n}=21) ; 3)$ excess alcohol intake (>20 g/day) $(\mathrm{n}=78) ; 4)$ cancer history ( $\mathrm{n}=14)$; and 5) BMI $<18.5 \mathrm{~kg} / \mathrm{m}^{2}$ $(\mathrm{n}=20)$. Ultimately, 451 subjects (mean age: $46.8 \pm 8.8$ years) were enrolled in the study.

\section{Thyroid hormone and laboratory measure-}

\section{ments}

Data were collected via self-reported questionnaires, anthropometric examinations, and laboratory tests. The questionnaires included medical history, alcohol consumption, and smoking. Anthropometric data consisted of height and weight. BMI was calculated as weight in kilograms divided by height in meters squared. Blood samples were obtained after 8 hours of overnight fasting and were used to measure fasting plasma glucose (FPG), HbA1c, total cholesterol (TC), low-density lipoprotein-cholesterol (LDL-C), high-density lipoprotein-cholesterol (HDL-C), triglycerides, aspartate aminotransferase (AST), alanine aminotransferase (ALT), gamma-glutamyltransferase (rGT), albumin, creatinine, and thyroid function. Thyroid hormones, such as T3, $\mathrm{TSH}$, and free T4 were measured using chemistry luminescence immunoassay with the same company equipment (Cobas series, Roche Diagnostic, Switzerland). A Cobas 8000 (Roche, 
Mannheim, Germany) was used to measure blood uric acid, TC, triglyceride, HDL-C, and LDL-C levels by using the enzymatic colorimetric test method.

Non-alcoholic fatty liver disease was defined as the presence of fatty liver disease, as determined by ultrasonography, in the absence of the following: hepatitis B or hepatitis C viral infection, excessive alcohol intake ( $>20 \mathrm{~g} /$ day), other liver disease, and medications known to cause fatty liver disease. The 451 subjects were divided into four groups: control $(n=216)$, subjects with obese $(n=94)$, subjects with NAFLD $(n=48)$, and subjects with obese + NAFLD $(n=93)$. Obesity was defined as $B M I \geq 25 \mathrm{~kg} / \mathrm{m}^{2}$.

\section{Definition of subclinical hypothyroidism and hypothyroidism}

Subclinical hypothyroidism was defined biochemically as normal serum free T4 in the presence of elevated TSH $(>4.2 \mathrm{mIU} / \mathrm{L})$. Overt hypothyroidism was defined as elevated TSH, usually above $10 \mathrm{mIU} / \mathrm{L}$, with subnormal free T4.

\section{Statistical analyses}

Data are expressed as means \pm SD or medians $\left(25^{\text {th }}-75^{\text {th }}\right.$ percentile $)$. Metabolic risk factors and other clinical characteristics were compared between subjects with and without $\mathrm{SCH}$ after follow-up. Mann-Whitney tests were used to compare continuous variables and chi-square exact tests were used to detect differences between groups. Spearman's correlation analysis was used to generate correlation coefficients be- tween TSH levels at follow-up and other factors. Multivariate Cox proportional hazard analysis was used to estimate hazard ratios (HRs) with 95\% confidence intervals (CIs) for the presence of SCH after follow-up. The presence of $\mathrm{SCH}$ after follow-up was compared among the four groups using $\mathrm{HRs}$ for $\mathrm{SCH}$, as estimated by Cox's proportional hazard analysis. The time scale for the analysis was the time since the basal examination until the onset of $\mathrm{SCH}$ after at least 4 years of follow-up or the last follow-up visit. Statistical analyses were performed using PASW 17.0 software (SPSS, Inc., Chicago, IL, USA), and $P$-values $<0.05$ were considered statistically significant.

\section{RESULTS}

During the mean follow-up of approximately 7 years $(6.8 \pm 1.4$ years $), 42$ of the 451 subjects (7.3\%) had SCH after follow-up, but no subjects developed overt hypothyroidism. The baseline characteristics of subjects with and without $\mathrm{SCH}$ after follow-up are shown in Table 1. Subjects with SCH after follow-up had significantly higher baseline TSH levels than those with normal thyroid function. There was no significant difference in BMI or the presence of NAFLD between the two groups (Table 1).

In total, 141 of the 451 subjects $(31.3 \%)$ were obese (BMI > $\left.25 \mathrm{~kg} / \mathrm{m}^{2}\right)$, and 189 (41.9\%) subjects had NAFLD at baseline. There was no difference between obese and non-obese subjects in baseline TSH levels $(2.24 \pm 0.1 .39$ vs. $2.01 \pm$ 
Table 1. Baseline characteristics of the subjects according to development of subclinical hypothyroidism (SCH)

\begin{tabular}{lccc}
\hline & $\begin{array}{c}\text { The subjects with SCH } \\
(\mathrm{N}=42)\end{array}$ & $\begin{array}{c}\text { The subjects without SCH } \\
(\mathrm{N}=409)\end{array}$ & $P$ \\
\hline Age (year) & $50.5 \pm 9.5$ & $46.3 \pm 8.5$ & 0.008 \\
Sex (male, \%) & $25(59.5 \%)$ & $256(62.6 \%)$ & 0.408 \\
BMI (kg/m2) & $23.9 \pm 2.9$ & $23.7 \pm 2.9$ & 0.720 \\
Smoking (current smoker) & $2(22.2 \%)$ & $35(30.4 \%)$ & 0.676 \\
Total cholesterol (mg/dL) & $192.5 \pm 34.1$ & $194.1 \pm 33.0$ & 0.770 \\
Triglyceride (mg/dL) & $90.5(62.5-168.8)$ & $104.0(73.0-157.5)$ & 0.875 \\
HDL cholesterol (mg/dL) & $53.4 \pm 12.5$ & $52.0 \pm 13.8$ & 0.505 \\
LDL cholesterol (mg/dL) & $120.2 \pm 28.9$ & $122.5 \pm 31.3$ & 0.620 \\
Fasting glucose (mg/dL) & $86.4 \pm 13.8$ & $85.4 \pm 16.6$ & 0.681 \\
Albumin (g/dL) & $4.64 \pm 0.23$ & $4.61 \pm 0.25$ & 0.503 \\
AST (U/L) & $22.3 \pm 12.6$ & $23.9 \pm 13.8$ & 0.428 \\
ALT (U/L) & $22.9 \pm 7.4$ & $22.3 \pm 8.5$ & 0.731 \\
rGT (U/L) & $19.5(11.8-33.3)$ & $23.0(14.0-37.5)$ & 0.352 \\
Urea (mg/dL) & $13.5 \pm 3.0$ & $14.1 \pm 3.9$ & 0.228 \\
Creatinine (mg/dL) & $0.86 \pm 0.17$ & $0.82 \pm 0.18$ & 0.226 \\
T3 (ng/dL) & $107.9 \pm 13.9$ & $109.5 \pm 17.4$ & 0.273 \\
Free T4 (ng/dL) & $1.27 \pm 0.19$ & $1.33 \pm 0.19$ & 0.064 \\
TSH (mlU/L) & $4.3 \pm 1.7$ & $1.8 \pm 0.9$ & $<0.001$ \\
NAFLD (\%) & $18(42.9 \%)$ & $24(41.8 \%)$ & 1.000 \\
Obese subject (\%) & $16(38.1 \%)$ & $125(30.6 \%)$ & 0.382 \\
\hline
\end{tabular}

Abbreviations: ALT, Alanine transaminase; AST, aspartate aminotransferase; BMI, body mass index; HDL, High-density lipoprotein; LDL, lowdensity lipoprotein; NAFLD, non-alcoholic fatty liver disease; rGT, gamma-glutamyltransferase; TSH, thyroid stimulating hormone

1.23, respectively, $P=0.081)$, free T4 levels $(1.33 \pm 0.22$ vs. $1.32 \pm 0.19 \mathrm{ng} / \mathrm{d}, P=0.702)$, or T3 levels $(111.37 \pm 20.8$ vs. $108.9 \pm 23.2 \mathrm{ng} / \mathrm{d}$, $P=0.255)$.

There was no difference between subjects with NAFLD and the control group in baseline TSH levels $(2.14 \pm 1.41$ vs. $2.03 \pm 1.19 \mathrm{mIU} / \mathrm{L}$, respectively, $P=0.378)$, free T4 levels $(1.32 \pm 0.19$ vs. $1.33 \pm 0.20 \mathrm{ng} / \mathrm{dL}, P=0.657$ ), or T3 levels $(110.95 \pm 18.6$ vs. $108.71 \pm 24.9 \mathrm{ng} / \mathrm{dL}, P=$ $0.273)$. In addition, there was no correlation between TSH levels at final follow-up and age, BMI, TC, FPG, or liver enzymes (Table 2).

After adjustment for age, sex, smoking, and baseline TSH, the risk of SCH was not associated with BMI or the presence of NAFLD at baseline (Table 3, 4). However, when the subjects were divided into four groups, obese subjects with NAFLD had a higher risk of SCH than control subjects (Table 5).

\section{DISCUSSION}

In this study, we investigated the effects of obesity and NAFLD on thyroid function and found that obese subjects with NAFLD had a higher risk of thyroid dysfunction than control individ- 
Table 2. Correlation between TSH level at final follow-up and metabolic risk factors

\begin{tabular}{lcc}
\hline \multirow{2}{*}{ Variable } & \multicolumn{2}{c}{ TSH } \\
\cline { 2 - 3 } Age (year) & Correlation coefficient & Pvalue \\
BMI (kg/m2) & 0.074 & 0.116 \\
Fasting glucose (mg/dL) & 0.033 & 0.484 \\
Total cholesterol (mg/dL) & 0.001 & 0.990 \\
Triglyceride $(\mathrm{mg} / \mathrm{dL})$ & -0.057 & 0.229 \\
LDL- cholesterol (mg/dL) & -0.010 & 0.836 \\
AST (U/L) & -0.003 & 0.955 \\
ALT (U/L) & 0.001 & 0.980 \\
GGT (U/L) & 0.064 & 0.175 \\
\end{tabular}

Abbreviations: BMI, body mass index

uals. Body composition is closely related to thyroid hormone. Thyroid hormones regulate basal metabolism and have an important role in lipid and glucose metabolism. ${ }^{16}$

Therefore, thyroid dysfunction is regarded as a cause of obesity and NAFLD. ${ }^{17,18}$ Thyroid dysfunction is associated with changes in body weight and body composition. Hypothyroidism is associated with a higher BMI and $\mathrm{SCH}$ is linked to significant changes in body weight. ${ }^{15}$ Even in euthyroid subjects, free T4 is inversely correlated with BMI. ${ }^{5,6}$ However, studies describing thyroid dysfunction caused by obesity also have been reported. In one study, serum TSH levels were higher in obese patients compared with the control group. ${ }^{12} \mathrm{~A}$ study by Marzullo et al. reported that obese patients had lower free T3 levels and free T4 levels, and obesity increased susceptibility to autoimmune thyroid disease. ${ }^{13}$ However, the exact mechanism underlying the thyroid function alterations in obesity is unknown.

Several explanations for the increased TSH lev- els observed in obesity have been suggested. The first is that they are caused by high leptin levels, since the subjects with obesity have a high leptin level and leptin stimulates pituitary TSH secretion. ${ }^{11}$

The second explanation is that they are due to increased deiodinase activity, serving as a defense mechanism in obese subjects by counteracting fat accumulation via increased energy expenditure. ${ }^{19}$ Another theory is that systemically increased adiposity is associated with steatosis in thyroid follicular cells, which may cause or influence the development of primary thyroid failure, since thyroid steatosis occurred in obese euthyroid patients. ${ }^{9}$ However, little is known about the effects of obesity on future thyroid function. Thus, we hypothesized that obesity or NAFLD at baseline may be associated with future thyroid dysfunction. In our study, there was no difference in free T4 or T3 levels at baseline between obese and non-obese subjects, unlike the study by Marzullo et al. ${ }^{13}$ The average BMI of the subjects in Marzullo et al $\left(42.9 \mathrm{~kg} / \mathrm{m}^{2}\right)$ was 
Table 3. Hazard ratios (HR) for presence of subclinical hypothyroidism according to presence of NAFLD

\begin{tabular}{lcc}
\hline \multicolumn{1}{c}{ Variable } & HR & $P$-value \\
\hline Crude Model HR & $(95 \% \mathrm{Cl})$ & 0.813 \\
$(95 \% \mathrm{CI})$ & $0.929(0.504-1.712)$ & \\
Adjusted HR ${ }^{*}$ & $1.229(0.615-2.454)$ & 0.559 \\
$(95 \% \mathrm{CI})$ & & \\
Model 1 & & \\
\hline
\end{tabular}

* by the multivariable Cox proportional hazard analysis

Abbreviations: NAFLD, non-alcoholic fatty liver disease

Multivariate model 1: adjusted for age, sex, smoking, baseline TSH

Table 4. Hazard ratios (HR) for presence of subclinical hypothyroidism according to presence of obesity

\begin{tabular}{lcc}
\hline \multicolumn{1}{c}{ Variable } & HR & $P$-value \\
\hline Crude Model HR & $195 \% \mathrm{Cl})$ & 0.260 \\
$(95 \% \mathrm{Cl})$ & $1.430(0.767-2.667)$ & 0.459 \\
Adjusted HR & & \\
$(95 \% \mathrm{CI})$ & $1.280(0.666-2.461)$ & \\
Model 1 & & \\
\hline
\end{tabular}

* by the multivariable Cox proportional hazard analysis

Multivariate model 1: adjusted for age, sex, smoking, baseline TSH

Table 5. Hazard ratios (HR) for presence of subclinical hypothyroidism in obese subjects with fatty liver

\begin{tabular}{lcc}
\hline \multicolumn{1}{c}{ Variable } & HR \\
$(95 \% \mathrm{Cl})$ & $P$-value \\
\hline Crude Model HR & $1.919(0.720-5.113)$ & 0.193 \\
$(95 \% \mathrm{Cl})$ & $3.203(1.138-9.014)$ & 0.027 \\
Adjusted HR & & \\
$(95 \% \mathrm{Cl})$ & & \\
Model 1 & & \\
\hline
\end{tabular}

* by the multivariable Cox proportional hazard analysis

Multivariate model 1: adjusted for age, sex, smoking, baseline TSH

different to that in the current study $\left(26.5 \mathrm{~kg} / \mathrm{m}^{2}\right)$, which may explain the different results.

In another study, obese subjects had moderately increased TSH levels without any thyroid disease. ${ }^{12}$ In the current study, there was no signifi- cant difference in TSH levels between the nonobese and obese groups $(2.01 \pm 1.24$ vs. $2.24 \pm$ 1.39 , respectively; $P=0.097$ ).

A few studies have investigated thyroid function and NAFLD. Some studies reported that TSH 
levels are higher in subjects with NAFLD, whereas others reported no association between TSH and NAFLD. ${ }^{17,18}$ The current study also showed that there was no difference in TSH levels between subjects with NAFLD and the control group. Furthermore, BMI and the presence of NAFLD at baseline were not related to future thyroid dysfunction (Tables 3, 4). However, a higher baseline TSH was related to the development of future thyroid dysfunction. When we divided the subjects into four groups according to obesity and the presence of NAFLD, obesity with NAFLD was related to future thyroid dysfunction (Table 5). A higher baseline TSH was also related to the development of future thyroid dysfunction.

Our study had several limitations. First, all of our subjects were volunteers undergoing a comprehensive health examination. Thus, they do not represent the general population. The prevalence of obesity in the study population was $31.3 \%$ and the prevalence of NAFLD was $41.9 \%$, both of which are higher than reported in the general population. Second, we included subjects with TSH levels $<10 \mathrm{mIU} / \mathrm{L}$ because the prevalence of thyroid dysfunction was very low when we included only patients with $\mathrm{TSH}<4.2 \mathrm{mIU} / \mathrm{L}$ after follow-up. So we included the subjects with TSH levels $<10 \mathrm{mIU} / \mathrm{L}$ and adjusted for baseline TSH in the multivariable Cox proportional hazard analysis, and the subjects with obese and NAFLD had a higher risk for the SCH than control subjects. Further studies are needed to evaluate the effects of obesity with ectopic steatosis on thyroid function. Third, we did not check for thyroglobulin antibody and thyroid peroxidase antibodies, which are associated with autoimmune thyroid disease. Finally, we could not demonstrate thyroid steatosis, and so we used NAFLD as marker of excessive lipid accumulation in non-adipose tissues.

In conclusion, this study showed that obese subjects with NAFLD at baseline was associated with thyroid dysfunction.

\section{ACKNOWLEDGEMENT}

This work (2015-04-001) was supported by the Fund for New Professor Research Foundation Program, Gyeongsang National University, 2015.

\section{REFERENCES}

1. Bedogni G, Miglioli L, Masutti F, Tiribelli C, Marchesini G, Bellentani S. Prevalence of and risk factors for nonalcoholic fatty liver disease: the Dionysos nutrition and liver study. Hepatology 2005;42:44-52.

2. Lazo M, Clark JM. The epidemiology of nonalcoholic fatty liver disease: a global perspective. Semin Liver Dis 2008;28:339-50.

3. Chung GE, Kim D, Kim W, Yim JY, Park MJ, Kim YJ, et al. Non-alcoholic fatty liver disease across the spectrum of hypothyroidism. J Hepatol 2012;57:150-6.

4. Sanyal D, Raychaudhuri M. Hypothyroidism and obesity: An intriguing link. Indian J En- 
docrinol Metab 2016;20:554-7.

5. Knudsen N, Laurberg P, Rasmussen LB, Bülow I, Perrild H, Ovesen L, et al. Small differences in thyroid function may be important for body mass index and the occurrence of obesity in the population. J Clin Endocrinol Metab 2005;90;4019-24.

6. Alevizaki M, Saltiki K, Voidonikola P, Mantzou E, Papamichael C, Stamatelopoulos K. Free thyroxine is an independent predictor of subcutaneous fat in euthyroid individuals. Eur J Endocrinol 2009;161:459-65.

7. Tagliaferri M, Berselli ME, Calò G, Minocci A, Savia G, Petroni ML, et al. Subclinical hypothyroidism in obese patients: relation to resting energy expenditure, serum leptin, body composition, and lipid profile. Obes Res 2001;19:196-201.

8. Szczepaniak LS, Victor RG, Mathur R, Michael D, Nelson MD, Szczepaniak EW, et al. Pancreatic steatosis and its relationship to $\beta$-cell dysfunction in humans: racial and ethnic variations. Diabetes Care 2012;35:237783.

9. Lee MH, Lee JU, Joung KH, Kim YK, Ryu MJ, Lee SE, et al. Thyroid dysfunction associated with follicular cell steatosis in obese male mice and humans. Endocrinology 2015;156:1181-93.

10. Kok P, Roelfsema F, Frölich M, Meinders AE, Pijl H. Spontaneous diurnal thyrotropin secretion is enhanced in proportion to circulating leptin in obese premenopausal women. J Clin Endocrinol Metab 2005; 90:6185-91.
11. Mantzoros CS, Ozata M, Negrao AB, Suchard MA, Ziotopoulou M, Caglayan S, et al. Synchronicity of frequently sampled thyrotropin (TSH) and leptin concentrations in healthy adults and leptin-deficient subjects: evidence for possible partial TSH regulation by leptin in humans. J Clin Endocrinol Metab 2001;86:3284-91.

12. Rotondi M, Leporati P, La Manna A, Pirali B, Mondello T, Fonte R, et al. Raised serum TSH levels in patients with morbid obesity: is it enough to diagnose subclinical hypothyroidism? Eur J Endocrinol 2009;160:403-8.

13. Marzullo P, Minocci A, Tagliaferri MA, Guzzaloni G, Di Blasio A, De Medici C, et al. Investigations of thyroid hormones and antibodies in obesity: leptin levels are associated with thyroid autoimmunity independent of bioanthropometric, hormonal, and weight-related determinants. J Clin Endocrinol Metab 2010;95:3965-72.

14. Rosenbaum M, Hirsch J, Murphy E, Leibel RL. Effects of changes in body weight on carbohydrate metabolism, catecholamine excretion, and thyroid function. Am J Clin Nutr 2000;71:1421-32.

15. Danforth E Jr, Horton ES, O'Connell M, Sims EA, Burger AG, Ingbar SH, et al. Dietary induced alterations in thyroid hormone metabolism during overnutrition. J Clin Invest 1979;64:1336-47.

16. Mullur R, Liu YY, Brent GA. Thyroid hormone regulation of metabolism. Physiol Rev 2014;94:355-82.

17. Tao Y, Gu H, Wu J, Sui J. Thyroid function 
is associated with non-alcoholic fatty liver disease in euthyroid subjects. Endocr Res 2015;40:74-8.

18. Zhang J, Sun H, Chen L, Zheng J, Hu X, Wang S, et al. Relationship between serum TSH level with obesity and NAFLD in euthyroid subjects. J Huazhong Univ Sci Technolog Med Sci 2012;32:47-52.

19. Reinehr T. Obesity and thyroid function. Mol Cell Endocrinol 2010;316:165-71. 